\title{
Late Presentation of Left Ventricular Outflow Tract Obstruction due to Subaortic Membrane
}

\author{
Giorgio Mastroiacovo ${ }^{1}$, Marco Gennari ${ }^{1}$, Sarah Ghulam Ali ${ }^{2}$, Gianluca Polvani ${ }^{3}$, and Marco \\ Agrifoglio 4 \\ ${ }^{1}$ Centro Cardiologico Monzino IRCCS \\ ${ }^{2}$ Centro Cardiologico Monzino Istituto di Ricovero e Cura a Carattere Scientifico \\ ${ }^{3}$ centro cardiologico monzino \\ ${ }^{4}$ Centro Cardiologico Monzino
}

April 28, 2020

\begin{abstract}
Left ventricular outflow tract (LVOT) obstruction is caused by a wide range of different anatomical lesions referred to as subaortic stenosis (SS). SS can be isolated or associated with other heart defects; and tends to evolve and progress over time even though the speed of its progression is very variable and difficult to predict. We present a case of a patient affected by sub aortic membrane undergone to surgical procedure.
\end{abstract}

\section{Text:}

Left ventricular outflow tract (LVOT) obstruction is caused by a wide range of different anatomical lesions, which are collectively referred to as subaortic stenois (SS). SS can be isolated or associated with other heart defects; in the most dramatic cases it can be part of a syndrome that includes several obstructive lesions of the left heart, called the Shone complex. Isolated SS can be caused by several factors: displacement of the mitral papillary muscle, displacement of the infundibular septum, abnormal insertion of the mitral valve or presence of exuberant fibrous tissue [1].

Subaortic stenosis tends to evolve and progress over time even though the speed of its progression is very variable and difficult to predict. In addition to the risk of progression, patients with subaortic stenosis face two other types of problems: an increased risk of infectious endocarditis and aortic valve insufficiency if the fibrous strends extend below the aortic valve cusps. The aortic valve may be normal or stenotic. Subaortic stenosis, leading to a LVOT obstruction, causes an increase in post-loadoing determining a concentrical ventricular hypertrophy, especially at the septal insertion of the membrane [1].

SS is usually suspected in young adults when the valve anatomy is not clearly stenotic, whereas Doppler examination reveals high transaortic pressure gradient [2].

Concerning the surgical treatment of SS, many dilemmas still remain open; it is uncertain which is the right timing and the type of procedure, although it has been noted that an early intervention decreases the risk of valve failure, even if the risk of stenosis recurrence remains high in the long term period.

We report images from a case of subaortic stenosis in a 72 years old woman referring to our Institution for exertional dyspnea (NHYA III). The patient was successfully operated of subaortic membrane removal and septal myectomy according to Morrow, the aortic valve was not surgically treated as it was neither stenotic nor insufficient (Fig.1). The course in the operating theatre was regular, the weaning from extracorporeal circulation did not require any inotropic support. Respiratory weanig and extubation occurred in the first 
few hours after arrival in the ICU. The rest of the hospitalization in intensive care and cardiac surgery ward was regular and free from major cradiovascular events. The patient was discharged to a rehabilitation center on the fifth post-operative days completely asymptomatic for angor and dyspnea (NYHA I) .

\section{Image Legend}

Fig 1. A: subaortic membrane at TEE; B: intraoperative image of the subaortic membrane; C and D: TEE after subaortic membrane removal.

\section{References}

1. Alain Serraf MD Joy Zoghby MD et al. Surgical treatment of subaortic stenosis: A seventeen-year experience The Journal of Thoracic and Cardiovascular Surgery Volume 117, Issue 4, April 1999, Pages 669-678.

2. Sylvestre Maréchaux, Françis Juthier et al. Illustration of the echocardiographic diagnosis of subaortic membrane stenosis in adults: surgical and live three-dimensional transoesophageal findings European Journal of Echocardiography, Volume 12, Issue 1, January 2011, Page E2
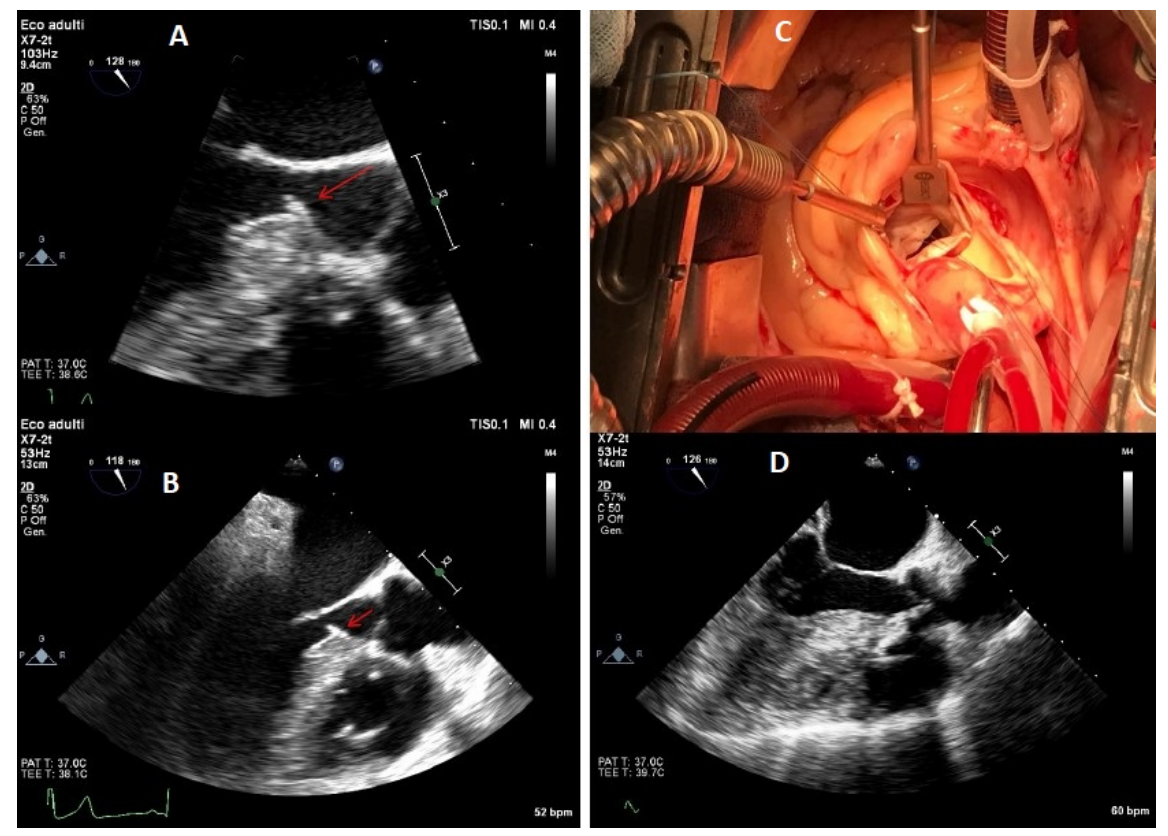